\title{
Characterization of Resist Molds and Replicated Nickel Dies for Fabricating Micro-Lens Arrays
}

\author{
Toshiyuki Horiuchi*, Ryunosuke Sasaki**, Keigo Komatsubara* and Hiroshi Ono** \\ *Mechanical Engineering, **Graduate school of Engineering, Tokyo Denki University, \\ 5, Senju-Asahi-cho, Adachi-ku, Tokyo, 120-8551, Japan \\ horiuchi@cck.dendai.ac.jp
}

\begin{abstract}
A new method for fabricating metal dies for the use of micro-lens array molding or stamping was proposed and investigated. As a metal, nickel was used, and the nickel dies were fabricated by replicating the primary resist molds that were directly printed using projection lithography on silicon wafers coated with approximately $10-\mu \mathrm{m}$ thick positive resist PMER P-LA900PM. Lens array patterns with quasi-spherical cross sections were printed by selecting a wavelength of $405-\mathrm{nm}$, defocuses between +100 and $+200 \mu \mathrm{m}$ and exposure times of 20-30 min. After printing resist mold patterns of a lens array, a thin gold film was spattered on it, and nickel was electroplated next. The aimed nickel dies were obtained by removing the resist molds from the electroplated nickel blocks. The diameters of nickel dies were faithfully corresponded to those of the resist molds. In addition, the surfaces of nickel dies were very smooth, and the peak-to-valley roughness was almost less than a quarter of visible light wavelengths. The new method will be promisingly effective for fabricating micro-lens arrays.
\end{abstract}

Keywords: resist mold, quasi-spherical pattern, nickel electroplating, nickel die,

\section{Introduction}

Various methods for fabricating micro-lens arrays have been proposed. In many cases, how to make the fist molds or the original lens array profiles is the most important subject. Thermal reflow of resist patterns is a most popular method [1-6]. Lithography using gray tone masks is another typical method $[7,8]$. In addition, etching methods [9-11], inkjet printing [12-14], and others [15-17] are also proposed.

Among such various methods, what methods are suitable depends on the size, curvature radius, lens pitch, and others. However, if which method is used, it is always strongly required that the surface profile curves are accurately controlled so as the light rays are refracted as being designed. Moreover, roughness of the lens mold surface should be sufficiently small comparing with the wavelengths of visible light so that the used light rays are not scattered nor irregularly reflected and refracted.
The authors have proposed another new method for fabricating micro-lens array molds of resist directly using projection lithography $[18,19]$. In the method, resist mold patterns are directly printed by a single lithography process only once. In spite of such easiness and simplicity, pattern profiles become quasi-spherical because the patterns are printed under the conditions of intentional very large defocuses and relatively long exposure times using exposure light rays with special wavelengths appropriately absorbed near the resist surface.

In this paper, it is shown in detail that the profiles of resist molds are favorably controlled by selecting the appropriate exposure wavelength, defocus and exposure dose. In addition, the resist molds are precisely replicated to nickel dies by thickly electroplating nickel on the resist mold patterns. Moreover, the nickel dies are certainly separated from the resist molds. Thus, it is 
demonstrated that the nickel dies are fabricated with high accuracies. Because the resist profiles are formed in room temperatures, the profiles do not change and deform during the patterning processes. As a result, almost sufficient accuracies generally required for metal dies of micro-lens arrays are obtained.

\section{Total Process Flow}

Main sequential processes for fabricating nickel dies proposed here are shown in Fig. 1. At first, mold patterns of the positive resist are formed on silicon wafers using projection lithography, as shown in (a) and (b). Next, wafer chips with the resist molds are fixed on copper plates using varnish, and the copper plates were used as cathodes of nickel electroplating. Thereafter, a gold thin film is spattered and deposited on the resist patterns, as shown in Fig. (c). Thus, the conductivity is given to the surfaces of resist patterns, and they become to work as a cathode of electroplating. Next, nickel is electroplated on them, as shown in (d). The thickly electroplated nickel is used as the dies after they are separated from the resist mold patterns, as shown in (e).

(a)

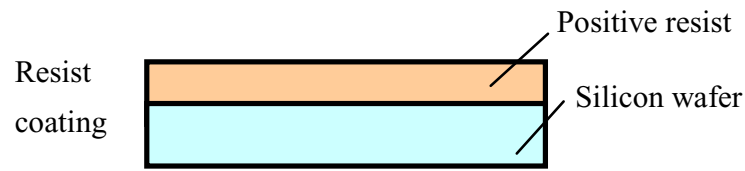

(b) Patterning

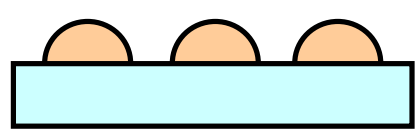

(c) Spattering

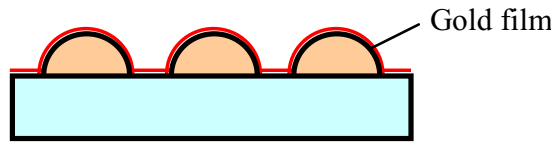

(d)

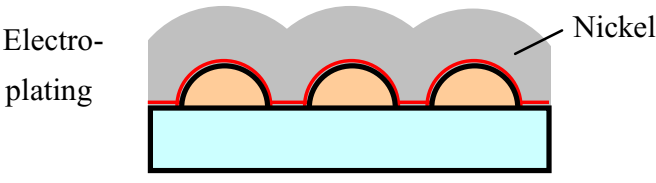

(e) Separation

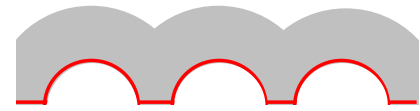

Fig. 1. Total process flow for fabricating resist molds and nickel dies.

\section{Characterization of Resist Molds}

\subsection{Fabrication of Resist Molds}

Quasi-spherical resist patterns suitable for using as lens array molds were printed directly using lens projection lithography. A handmade exposure system was used for printing the patterns, as shown in Fig. 2. As a projection lens, a versatile camera lens (Canon New FD 28/2.8) was used. The reduction ratio was approximately $1 / 19$, and the numerical aperture was controlled to approximately 0.07 by placing an aperture plate with a diameter of $4 \mathrm{~mm}$ on the camera lens. As an exposure light source, a special ultra violet lamp (Sumita Optical Glass LS-140UV) was used. The light emitted from the lamp contained wavelength components between 250 and $700 \mathrm{~nm}$. However, to print patterns with quasi-spherical cross sections surely, light rays with wavelengths of approximately 405 $\mathrm{nm}$ were selectively used by inserting a band path filter with a full width half maximum (FWHM) of $8 \mathrm{~nm}$ (Asahi Spectra MZ0405).

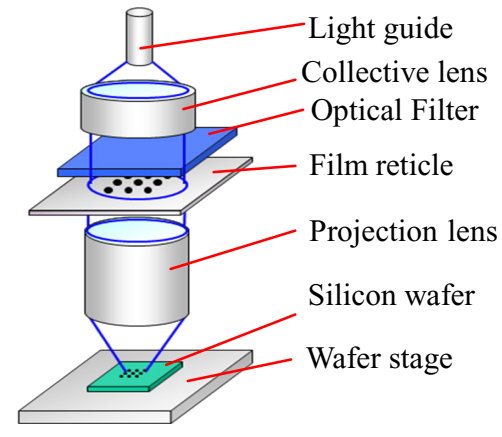

(a) Schematic figure of exposure system.

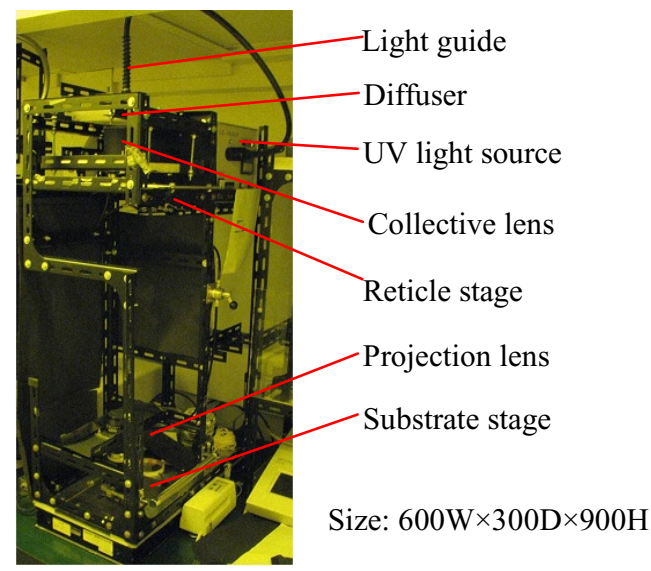

(b) Outside view of exposure system.

Fig. 2. Exposure system used for this research. 
On the other hand, flexible film reticles were used because they were inexpensive and easily prepared in short turn-around times.

As a resist, positive PMER LA-900PM (Tokyo Ohka Kogyo) was used, and it was coated on silicon wafers in approximately $10 \mu \mathrm{m}$ thick. After the exposure, the resist was developed in PMER P-7G (Tokyo Ohka Kogyo) for 4 min. P-7G is a set developer of the resist. The main component of the resist is a novolac resin, and the developer is a $3 \%$ aqueous solution of Tetra-methyl-ammoniumhydro-oxide (TMAH).

There are three important parameters for controlling the cross section profiles of resist patterns. The first parameter is the exposure wavelength or the absorption of exposure light into the resist material. The light energy $E_{d}$ at the depth of $d$ from the resist surface is expressed as

$$
E_{d}=E_{s} e^{-\alpha d} .
$$

Here, $E_{s}$ is the light energy at the surface, and $\alpha$ is the absorption coefficient of the resist material. For this reason, $E_{d}$ decreases according to the increase of $\alpha$ value. As a result, exposure light rays are much absorbed near the resist surface, and shoulders of positive resist patterns are rounded. Fig. 3 compares cross section profiles of line-and-space patterns printed by exposure light rays with wavelengths of 405 and $436 \mathrm{~nm}$. Using 405-nm light, shoulders of patterns became notably round even if the focal position of the wafer was carefully adjusted so that the sidewalls became as vertical as possible.

The second parameter is the focal position or the defocus. Cross section profiles of resist patterns gradually approach to semispherical ones by defocusing positions of pattern images in the direction that wafers are moved far from the lens,

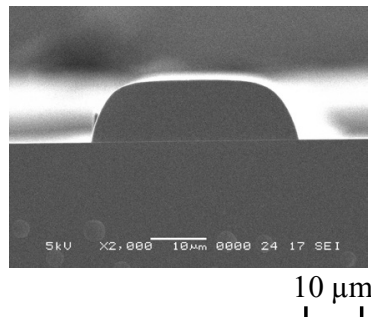

(a) $\gg=405 \mathrm{~nm}$

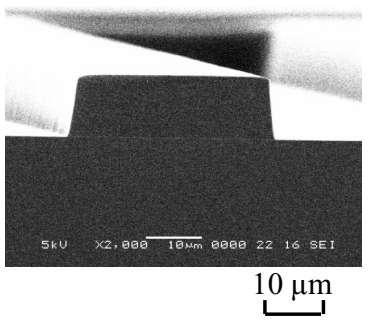

(b) $\lambda=436 \mathrm{~nm}$
Fig. 3. Pattern profile dependence on wavelength.

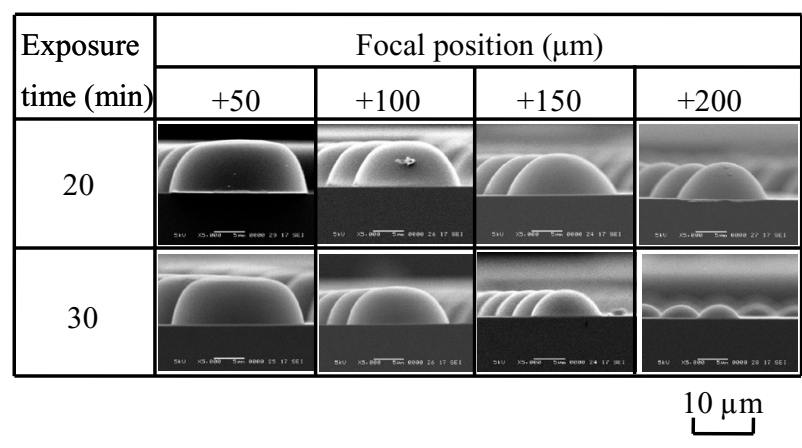

Fig. 4. Pattern profile dependence on focal position.

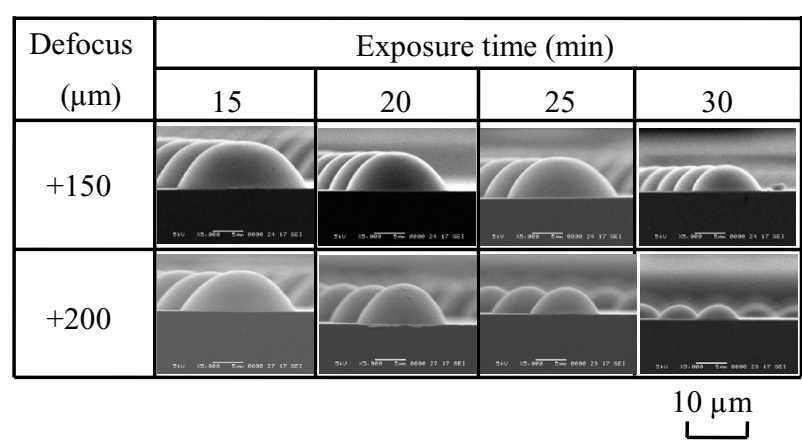

Fig. 5. Pattern profile dependence on exposure time.

as shown in Fig. 4. The outline contours of cross sectional profiles became almost circular at the defocuses between +100 and $+200 \mu \mathrm{m}$. Here, plus marks mean that wafers were moved in the above mentioned direction.

The third parameter is the exposure time. Heights of the quasi-spherical patterns were gradually reduced by increasing the exposure time, as shown in Fig. 5.

\subsection{Evaluation of Resist Molds}

Considering above mentioned patterning characteristics, accuracies of lens pattern profiles were evaluated using resist patterns printed by the defocus of $+100 \mu \mathrm{m}$ and exposure time of $30 \mathrm{~min}$. First, outside views and top-view diameters of lens array molds were evaluated. Fig. 6 shows panorama top view of lens array molds. It was demonstrated that the molds were smoothly and neatly fabricated. The homogeneity of top-view diameters were evaluated at $4 \times 4=16$ points in the center area of the array where the image intensities were considerably homogeneous. The lens pitch in the array was $52.6 \mu \mathrm{m}$, and top-view diameters were measured by the distance interval of 5 pitches. 
Accordingly, the distances between measured neighbor points in the line and column directions were $263 \mu \mathrm{m}$, respectively. Therefore, the evaluated area size was almost $0.8 \times 0.8 \mathrm{~mm}^{2}$. Examples of top-view photographs used for diameter measurements are shown in Fig. 7. The diameters of patterns were measured in four directions in each Figure, and averaged. The average diameters were plotted in Fig. 8. It was

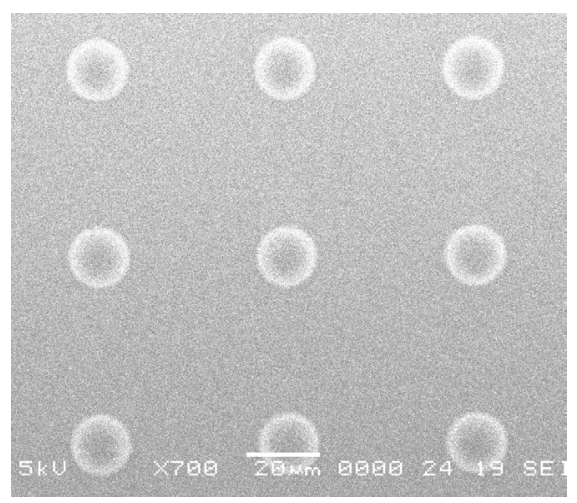

Fig. 6. Panorama top view of resist mold patterns.
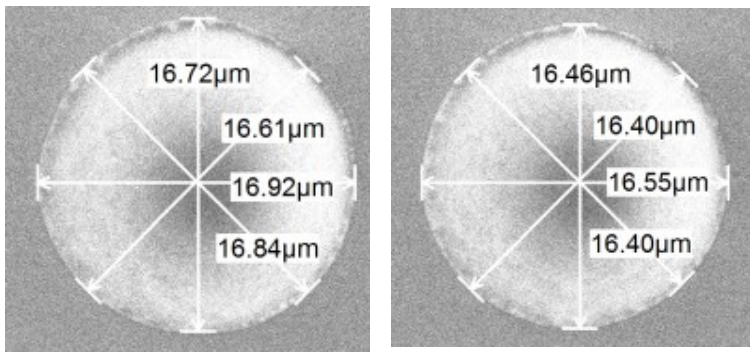

Fig. 7. Diameter measurement of resist mold patterns.

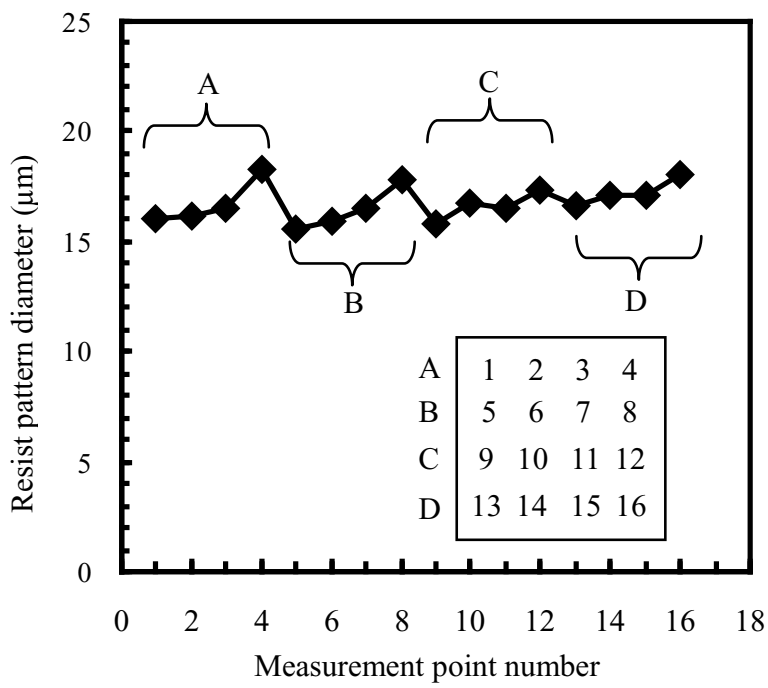

Fig. 8. Diameter distribution of resist mold patterns. clarified that top-view diameters were almost homogeneous. Remained diameter fluctuation was mainly caused by the illumination non-uniformity of the exposure light.

\section{Characterization of Nickel Dies}

\subsection{Replication of Nickel Dies}

After printing quasi-spherical resist patterns, the pattern profiles were replicated to metal dies. As a metal, nickel was used because it was easily electroplated on various materials. Before the electroplating, each wafer chip was fixed on a copper plate using varnish. Next, a thin gold film was spattered on them to give the conductivity to the resist patterns, As a spattering system, the one prepared for observing specimens using a scanning electron microscope was used. (Sanyu Electron SC-701)

Nickel was electroplated in an aqueous solution of nickel amidosulfate, as shown in Fig. 9. Nickel amidosulfate of $135 \mathrm{gf}$, nickel chloride of $0.9 \mathrm{gf}$, boric acid of $9 \mathrm{gf}$, and sodium dodecylsulfate of $0.15 \mathrm{gf}$ were dissolved in $300 \mathrm{ml}$ of water. The resist molds fixed on the copper plate and covered by the gold thin films were totally used as a cathode by soldering a lead wire, and nickel bars are used as anodes. The electroplating speed was controlled by changing the voltage applied between the anode and cathode. The thickness of electroplated nickel was controlled by setting the electroplating time for $300 \mathrm{~min}$ by keeping the current density at $30 \mathrm{~A} / \mathrm{dm}^{2}$ and the temperature at $40-50^{\circ} \mathrm{C}$ constant. Under these conditions, nickel thickness became approximately $130 \mu \mathrm{m}$.

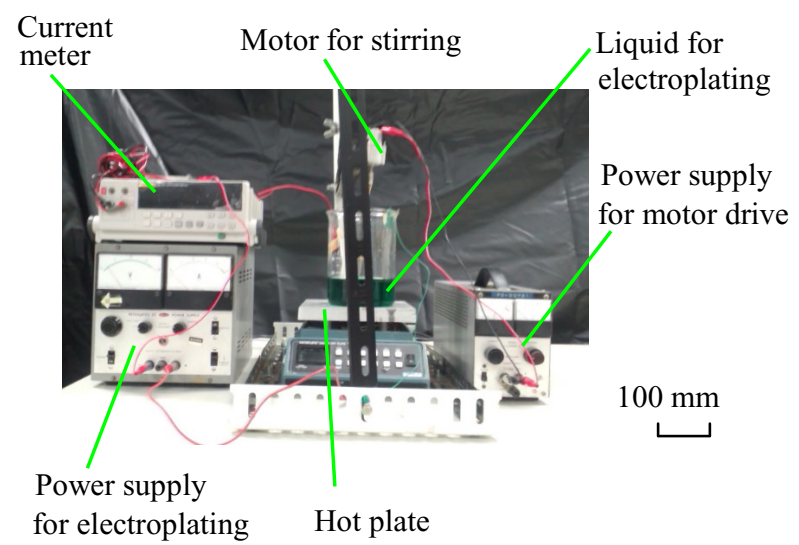

Fig. 9. Setup for electroplating nickel. 
The nickel dies were mechanically separated from the resist molds. However, fragments of resist molds were partially remained on the nickel dies. For this reason, they were removed by dissolving chemically. Thus, aimed nickel dies were obtained.

\subsection{Evaluation of Nickel Dies}

Accuracies of replicated nickel dies were evaluated by measuring the top-view diameters. Fig. 10 shows panorama top view of nickel dies. It was verified that the nickel dies were successfully fabricated also. Examples of top-view photographs used for diameter measurements are shown in Fig. 11. The top-view diameters were measured in four directions, as shown in the Figure. Fig. 12 shows the distribution of nickel die diameters in $0.8 \times 0.8$ $\mathrm{mm}^{2}$ field, the selfsame as the one used for the evaluation of resist mold patterns. The nickel die diameter should be the same as the resist mold diameter if they are not deformed by the electroplating. For this reason, differences between the diameters of nickel dies and resist molds were investigated, as shown in Fig. 13. It was clarified that both diameters were almost exactly same. The deviations were within $\pm 300 \mathrm{~nm}$.

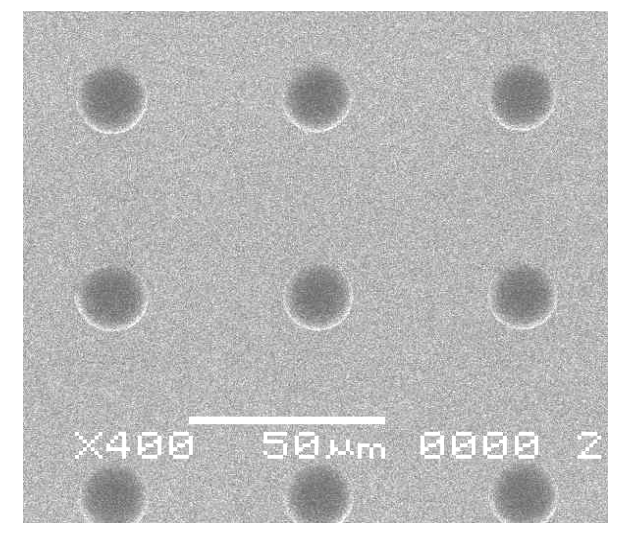

Fig. 10. Panorama top view of nickel dies.
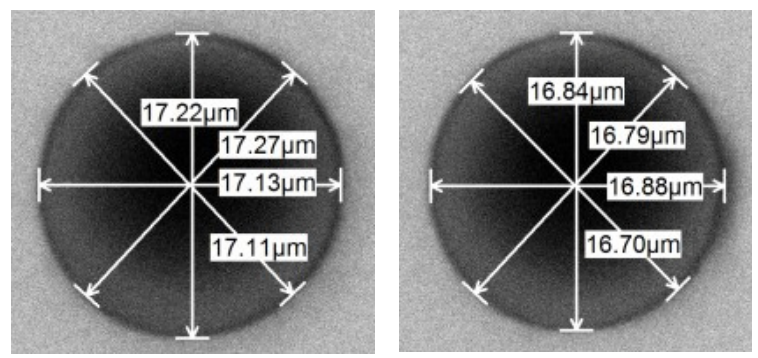

Fig. 11. Diameter measurement of nickel dies.

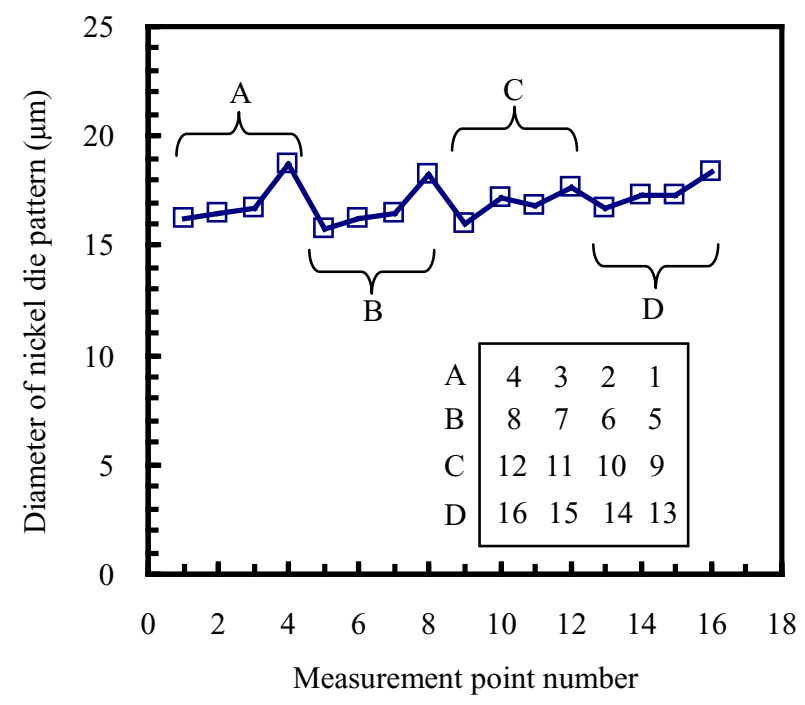

Fig. 12. Diameter distribution of nickel die patterns.

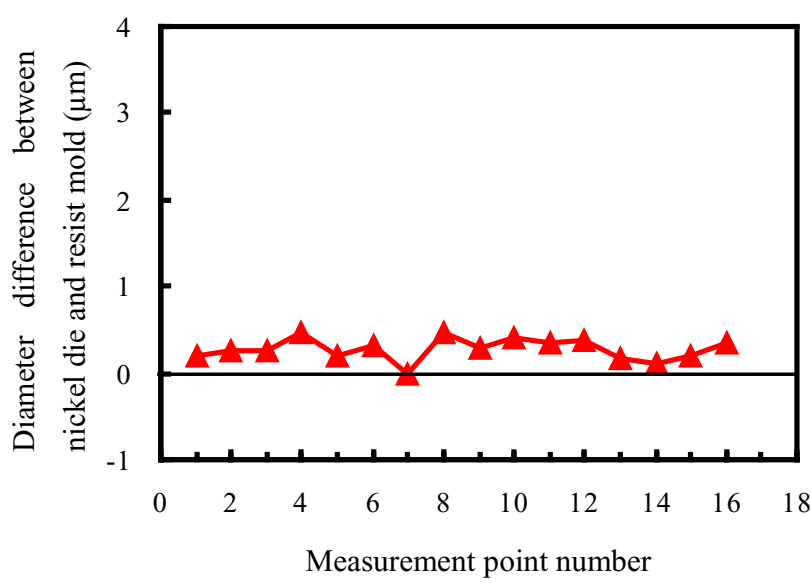

Fig. 13. Diameter differences between the resist mold and nickel die patterns

Next, the surface smoothness of a nickel die was investigated. Fig. 14 shows a contour curve of a concave nickel die element. The curve was obtained using a laser microscope (Keyence VK-8510). It was verified that the maximum peak-to-valley roughness was less than $100 \mathrm{~nm}$, and the roughness was smaller than a quarter of the

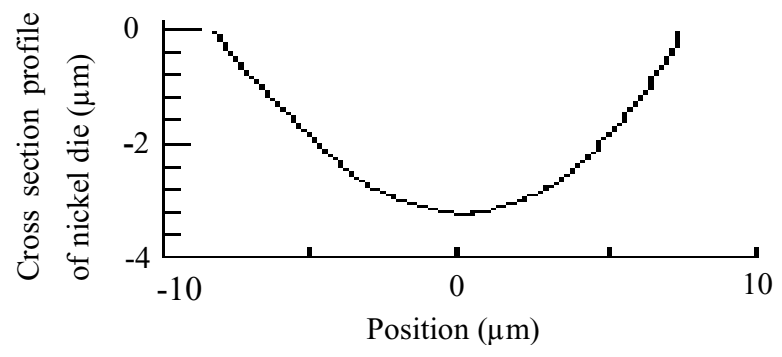

Fig. 14. Smooth contour of nickel die patterns. 
central wavelength of visible light. Accordingly, it is considered that the nickel dies are applicable to the fabrication of micro-lens arrays using hot-stamping or injection-mold methods.

\section{Conclusion}

A new method for fabricating resist molds and nickel dies of micro-lens arrays was proposed, and the feasibility of the method was investigated. Resist mold patterns with quasi-spherical cross sectional profiles were directly printed on silicon wafers by selecting the appropriate exposure wavelength of $405 \mathrm{~nm}$, large defocus between $+100 \mu \mathrm{m}$ and $+200 \mu \mathrm{m}$ in the light path direction, and giving sufficient exposure doses. Nickel dies were fabricated by electroplating nickel thickly on the resist molds covered by thin spattered gold films. The profiles were faithfully replicated, and the replication errors of top-view diameters were less than $\pm 300 \mathrm{~nm}$. Moreover, the surface roughness of a finished nickel die was less than a quarter of the central wavelength of visible light. Thus, it was demonstrated that metal dies were precisely fabricated using the proposed method.

\section{Acknowledgement}

This work was partially supported by Research Institute for Science and Technology of Tokyo Denki University, Grant Number Q13T-02.

\section{References}

1. M. C. Chou, C. T. Pan, S. C. Shen, M. F. Chen, K. L. Lin and S. T. Wu, Sensors and Actuators, A 118 (2005) 298.

2. C. T. Pan, C. H. Su, Sensor and Actuators A 134 (2007) 631.

3. C. T. Pan, T. T. Wu, M. F. Chen, Y. C. Chang, C.J. Lee and J. C. Huang, Sensors and Actuators, A
141 (2008) 422.

4. J. C. Tsai, M. F. Chen and H. Yang, DTIP 2008, EDA Publishing, Apr. (2008) 174.

5. S. S. Oh, C. G. Choi and Y. S. Kim, Microelectron. Eng. 87 (2010) 2328.

6. K. H. Liu, M. F. Chen, C. T. Pan, M. Y. Chang and W. Y. Huang, Sensors and Actuators, A 159 (2010) 126.

7. J. Yao, Z. Cui, F. Gao, Y. Zhang, Y. Guo, C. Du, H. Zeng and C. Qiu, Microelectron. Eng. 57-58 (2001) 729

8. J. J. Yang, Y. S. Liao and C. F. Chen, Optics Communications 270 (2007) 433.

9. C. S. Lim, M. H. Hong, A. S. Kumar, M. Rahman, X. D. Liu, Int. J. Machine Tools \& Manufacture 46 (2006) 552.

10. F. C. M. J. M. Delft, C. Ebm, E. P. Naburgh, E. Platzgummer, H. Loescher and G. Gross, Microelectron. Eng., 85 (2008) 937.

11. H. Oh, G. Kim, H. Seo, Y. Song, K. Lee and S. S. Yang, Sensors and Actuators, A 164 (2010) 161.

12. L. Yulin, L. Tonghai, J. Guohua, H. Junmin and W. Lili, Optik, 118 (2007) 395.

13. A. Voigt, U. Ostrzinski, K. Pfeiffer, J. Y. Kim, V. Fakhfouri, J. Brugger and G. Gruetzner, Microelectron. Eng. 88 (2011) 2124.

14. H. S. Lee, I. Park, K. S. Jeon, E. H. Lee, Microelectron. Eng. 87 (2010) 1447.

15. B. K. Nguyen, E. Iwase, K. Matsumoto and I. Shimoyama, MEMS 2007, Jan. (2007) 305.

16. T. K. Shih, C. F. Chen, J. R. Ho and F. T. Chuang, Microelectron. Eng. 83 (2006) 471.

17. I. S. Kang, J. S. Kim, M. C. Kang and K. Y. Lee, J. Materials Processing Technol. 20I (2008) 585.

18. H. Ono and T. Horiuchi, Jpn. J. Appl.Phys. 51 (2012) 06FL20.

19. T. Horiuchi and H. Ono, Dig. Abst. Book, IEEE 12th Int. Conf. Nanotechnol. Aug. (2012) 169. 